\title{
Perfil epidemiológico da COVID-19 nos países da América Central Continental: análise retrospetiva do primeiro semestre de 2020
}

\author{
Roberth Steven Gutiérrez-Murillo' \\ Lina Lizeth Leal-Ospina ${ }^{2}$ \\ Andrea Del Pilar Trujillo-Rodríguez ${ }^{3}$
}

\section{RESUMO}

Considerando o panorama latino-americano de emergência de saúde pública causada pela pandemia da COVID-19, tornase oportuno resgatar o olhar para o eixo centro-americano, no intuito de contribuir com o atual conhecimento sanitarista para o controle e prevenção das doenças infecciosas de interesse público. O presente estudo tem por objetivos traçar o perfil sociossanitário e descrever comportamento epidemiológico da COVID-19 nos países da América Central Continental, durante o primeiro semestre do ano de 2020.

Termos de indexação: Infeccção por coronavírus; Pandemia; Saúde Pública; América Latina.

\section{ABSTRACT}

Considering the Latin American panorama of public health emergency caused by the COVID-19 pandemic, it is opportune to take a look at the Central American axis, in order to contribute with the current sanitary knowledge to the control and prevention of infectious diseases of public interest. The present study aims at outlining the socio-sanitarian profile and describing the epidemiological behavior of COVID-19 in the countries of Continental Central America during the first half of 2020 .

Indexing Terms: Coronavirus infection; Pandemic; Public Health; Latin America.

\footnotetext{
${ }^{1}$ Mestrando no Programa de Pós-graduação em Gerontologia pela Universidad Europea del Atlántico, Cantábria, Santander, Espanha. Sanitarista Residente no Programa de Residência Multiprofissional em Saúde da Família e Graduado em Saúde Coletiva pela Universidade Federal da Integração Latino-Americana, Foz do Iguaçu, Paraná, Brasil.

2Bacharelanda em Geografia pela Universidade Federal da Integração Latino-Americana, Foz do Iguaçu, Paraná, Brasil.

${ }^{3}$ Mestranda no Programa de Pós-graduação em Saúde Pública pela Universidade de São Paulo, São Paulo, Brasil. Graduada em Saúde Coletiva pela Universidade Federal da Integração Latino-Americana, Foz do Iguaçu, Paraná, Brasil.
}

\section{Como citar este artigo / How to cite this article}

Gutiérrez-Murillo RS, Leal - Ospina LL, Trujillo - Rodriguez Andrea DP. Perfil epidemiológico da COVID-19 nos países da América Central Continental: análise retrospetiva do primeiro semestre de 2020. InterAm J Med Health 2020;3:e202003063. 


\section{INTRODUÇÃO}

O ano de 2019 estabeleceu o início de uma nova emergência sociossanitária a nível mundial. Em dezembro de 2019, na cidade de Wuhan, província de Hubei, China, notificou-se a mais recente manifestação virológica do coronavírus, logo nomeado de SARS-CoV-2 e, em seguida, decretada pela Organização Mundial da Saúde (OMS) a condição de alcance pandêmico [1]. O SARS-CoV-2 é a causa primária para o diagnóstico da doença da COVID-19, a qual se caracteriza pela presença de um quadro agudo respiratório, espalhado por vários mecanismos, tais como uma desaceleração do metabolismo celular ou secreção de citocinas, comprometedoras da capacidade respiratória e da capacidade adaptativa do sistema imunológico [2].

Estudos epidemiológicos têm apontado maior coeficiente de letalidade em indivíduos com registro de comorbidades, especialmente aquelas que acometem os sistemas respiratório e circulatório, uma vez que os pulmões parecem serem os órgãos mais afetados [1,3]. Sendo que a idade avançada está relacionada com superior prevalência de doenças crônicas não-transmissíveis, principalmente cardiovasculares e respiratórias, pensa-se que os idosos constituem o grupo etário mais suscetível à doença [3]. Contudo, admite-se que o comportamento epidemiológico mundial projeta igual risco de contágio em todos os segmentos populacionais.

Na América Latina, os efeitos da COVID-19 têm sido visualizados não somente no âmbito hospitalar (haja vistas do alto coeficiente de morbimortalidade), uma vez que importantes desafios no cenário político, econômico e social têm requisitado da intervenção imediata dos governos. Citando, por exemplo, as previsões estatísticas divulgadas pela Comissão Econômica para a América
Latina e o Caribe (CEPAL) informam que a região latinoamericana sofrerá uma queda econômica de $9.1 \%$ no seu Produto Bruto Interno (PBI), a finais de 2020. Em relação ao istmo centro-americano, planteou-se uma tendência negativa de $6.2 \%$ no $\mathrm{PBI}$ [4]. Como efeitos imediatos no âmbito da integração centro-americana, a COVID-19 provocou a descontinuação de cadeias regionais de valor, o aumento do teletrabalho (Work From Home) e a automatização do comércio eletrônico. Ademais, os países entraram no consenso de fechar suas fronteiras e restringir o transporte (aéreo, terrestre e naval), o que desencadeou, consequentemente, a imposição de restrições nas exportações de insumos, equipamentos médicos e de alimentos [4].

Considerando o panorama latino-americano de emergência de saúde pública causada pela pandemia da COVID-19, torna-se oportuno resgatar o olhar para o eixo centro-americano, no intuito de contribuir com o atual conhecimento sanitarista para o controle e prevenção das doenças infecciosas de interesse público.

Geograficamente falando, a América Central Continental (ACC) é a sub-região com menor densidade populacional e extensão territorial $(523.000 \mathrm{Km} 2)$ do Continente Americano, conformada por sete nações, a saber: Belize, Costa Rica, El Salvador, Guatemala, Honduras, Nicarágua e Panamá. Sua localização a converte em uma ponte entre as regiões Sul e Norte do Continente, sendo o espanhol o idioma predominante [5]. O acesso à saúde, às tecnologias de saúde e especialmente aos medicamentos essenciais, ainda está fora do alcance da população em muitos países centro-americanos, e isso é agravado pelas desigualdades de acesso entre regiões e entre diferentes grupos populacionais [6].

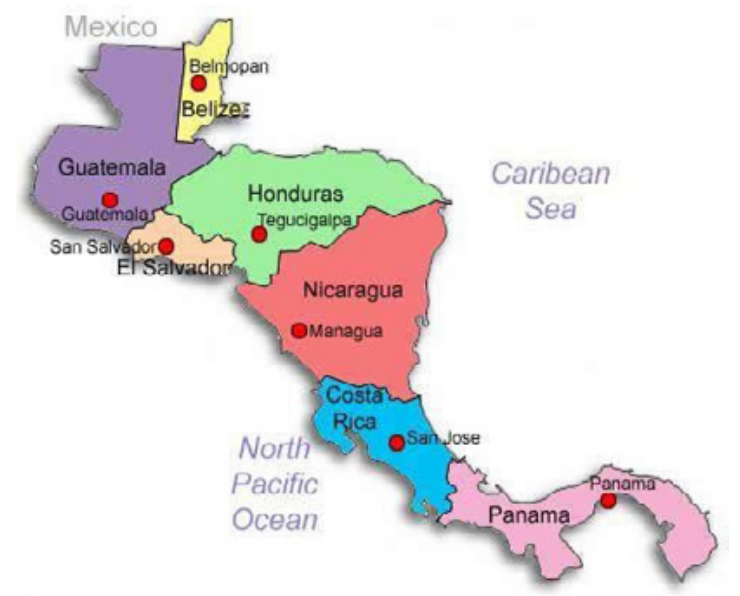

Figura 1. Extensão territorial da América Central Continental, segundo país e capital. Fonte: Adoptado de Só Geografia (2007) [7]. 
Com as amplas limitações sociossanitárias $[5,6]$ que caracterizam a maioria desses países, o presente trabalho pondera a hipótese de que os países com perfil sociossanitário menos favorável para o desenvolvimento humano (condições sanitárias, sociais e econômicas) serão aqueles com maior coeficiente de letalidade e contágio para o vírus.

Portanto, surge o interesse em traçar o perfil sociossanitário e descrever comportamento epidemiológico da COVID-19 nos países da ACC, durante o primeiro semestre do ano de 2020.

\section{MÉTODOS}

\section{Tipo de estudo}

Trata-se de um estudo ecológico misto (de séries temporais e de múltiplos grupos), de cunho analíticodescritivo e com viés quantitativa. Foram considerados todos os países que conformam o eixo continental centroamericano como unidades de estudo.

Hipótese de pesquisa

H0: $\mu$ = países com melhor (mais favorável) perfil de desenvolvimento humano apresentaram menor coeficiente de letalidade e de contágio pela COVID-19 durante o primeiro semestre de 2020;

$\mathrm{H} 1: \mu \neq$ países com pior (menos favorável) perfil de desenvolvimento humano apresentaram maior coeficiente de letalidade e de contágio pela COVID-19 durante o primeiro semestre de 2020 .

\section{Perfil Sociodemográfico}

Como variável dependente observou-se o perfil nacional social de cada país, e como variáveis independentes tomaram-se as subunidades: 1) estrutura demográfica (população total, taxa bruta de natalidade, taxa bruta de mortalidade e expectativa de vida); 2) educação (gasto público como \% do PIB); 3) saúde (razão da mortalidade materna, gasto público como \% do PIB); 4) pobreza (Coeficiente de Gini); 5) serviços básicos (proporção da população que utiliza fontes de água potável melhoradas e proporção da população que utiliza instalações sanitárias melhoradas) e 6) social (gasto público como \% do PIB).

Todos os dados eram de origem secundária, disponibilizados de forma gratuita e de acesso imediato por meio de consulta à Base de Dados e Publicações Estatísticas da CEPAL (CEPALSTAT) [8].

\section{Perfil Epidemiológico}

Como variável dependente contabilizou-se o total de casos confirmados para COVID-19 (infecções), e como variável independente o total de óbitos (fatalidades), durante de 01 de fevereiro a 31 de julho de 2020.

Todos os dados foram de origem secundária, disponibilizados de forma gratuita e de acesso imediato por meio de consulta on-line ao painel virtual COVID-19 Projections, do Institute of Health Metrics and Evaluation (IHME) - University of Washington School of Medicine [9].

\section{Instrumento e coleta de dados}

A extração dos dados foi realizada por meio de consulta aos sítios referidos para cada perfil, tais foram: CEPALSTAT e IHME $[8,9]$, durante o mês de agosto, de 2020.

Foi elaborado um roteiro estruturado, que continha dados referentes ao perfil sociodemográfico dos países (variáveis dependente e independentes) e ao perfil epidemiológico da COVID-19 (variáveis dependente e independente), através do software Microsoft Excel®, de livre licença para iOS, no qual foram agrupados e tabulados os dados.

\section{Análise dos dados}

Os dados processados foram analisados por meio da abordagem quantitativa, empregando à técnica da estatística descritiva, com utilização de medidas de dispersão (média, desvio padrão, limite inferior, e limite superior), por meio do software PAST®, de livre licença para iOS. Os perfis regionais da ACC foram calculados por meio da somatória de todos os valores em cada variável (dependente e independente), dividido sobre o número total de países.

Para o cálculo do coeficiente de letalidade foi dividido o número de óbitos pela COVID-19 entre o número de indivíduos contagiados pela doença, multiplicado por 100. Dito coeficiente é de grande importância para a Saúde Pública, pois dá ideia da gravidade da doença, pois indica o percentual de pessoas que morreram por tal enfermidade/agravo, podendo informar sobre a qualidade da assistência médica oferecida à população [10]. Por outro lado, o coeficiente de contágio individual (para cada país) foi calculado pela divisão entre o total de casos confirmados por COVID-19 segundo país e o total de casos confirmados na ACC, multiplicado por 100. As informações foram apresentadas por meio de gráficos e 
tabelas comparativas, com valores absolutos (f), e relativos (\%).

\section{RESULTADOS}

A ACC apresenta importantes variações sociodemográficas, de nação a nação, conforme apresentado na Tabela 01. Em primeiro lugar, observouse uma população total de $50.739,000$ habitantes $(X=7.248,000, S=5.507,000)$, com projeções para $2020 / 25$. A Guatemala foi o país mais populoso $(35,3 \%)$ e Belize o que menos habitantes registrou (0.8\%).

A taxa bruta de natalidade regional correspondeu a $18.4 \%$, sendo expressivamente menor na Costa Rica $(12.7 \%)$ e superior na Guatemala $(23.0 \%)$, o que poderia inferir que a Costa Rica é o país centro-americano continental que mais rápido envelhecerá. Viu-se uma taxa bruta de mortalidade regional de $5.3 \%$, sendo unicamente ultrapassada por Costa Rica (5.4\%) e El Salvador (7.2\%).

$\mathrm{Na}$ análise da expectativa de vida, notou-se idade regional média de 76 anos, contudo, percebeu-se que apenas Costa Rica, Panamá e Honduras conseguiram alcançar dita média, com 81, 79 e 76 anos, respectivamente. Tais estatísticas reforçam a ideia de que a Costa Rica é o país com população mais velha na região.

Tabela 01. Perfil sociodemográfico da América Central Continental, segundo CEPALSTAT.

\begin{tabular}{ccccc}
\hline País & População(Hab)* & TBN(\%)** & EVG(anos)* & TBM(\%)* \\
\hline Belize & 398,000 & 19.6 & 75 & 4.9 \\
\hline Costa Rica & $5.094,000$ & 12.7 & 81 & 5.4 \\
\hline El Salvador & $6.486,000$ & 17.2 & 74 & 7.2 \\
\hline Guatemala & $17.916,000$ & 23.0 & 75 & 4.7 \\
\hline Honduras & $9.905,000$ & 20.3 & 76 & 5.5 \\
\hline Nicarágua & $6.625,000$ & 18.7 & 75 & 5.3 \\
\hline Panamá & $4.315,000$ & 17.9 & 79 & 5.3 \\
\hline MRAcc & $\mathbf{7 . 2 4 8 , 0 0 0}$ & 18.4 & 76 & 5.1 \\
\hline
\end{tabular}

Fonte: Elaborado pelos autores (2020).

O perfil sociossanitário da ACC é mostrado na Tabela 02. De acordo com os dados observados sobre o investimento estatal do PIB em educação, notou-se uma média regional de $5.0 \%$, sendo relativamente inferior na Guatemala (2.8\%), Panamá (3.2\%) e El Salvador (3.8\%). Em contraste a isso, viu-se que os países da Costa Rica e Belize investem $+2.4 \%$ do esperado.

A média regional de investimento estatal do PIB em saúde correspondeu a 7.0\%, não sendo alcançada pela Guatemala (5.7\%), Belize (6.2\%) e El Salvador (6.9\%). Chama a atenção que, no caso da Costa Rica, esse investimento supera em $1 \%$ o valor esperado. A razão de mortalidade materna na ACC foi de 60 óbitos entre cada mil mulheres. Sobressai que na Costa Rica e Belize esse valor é de apenas 27/000 e 36/000, respectivamente. Países como Nicarágua (98/000) e Guatemala (95/000) ainda apresentam importantes desafios na atenção integral à saúde da mulher.

Viu-se que, em média, a ACC expressou um Coeficiente de Gini igual a 0.48 até 2018, sendo que El Salvador indicou maior desigualdade social na distribuição da renda (0.41). Ademais, quando analisado o investimento 
estatal do PIB em estrutura social, notou-se uma média regional de $12.25 \%$, a qual foi duas vezes maior na Costa Rica $(24.0 \%)$ e estreitamente inferior nas nações de Guatemala (7.0\%), Honduras (8.0\%) e Panamá (8.8\%). Não foi possível incluir dados do setor social em Belize, uma vez que o país não registrou estatísticas na CEPALSTAT, desse modo, os valores informados nesse quesito (\% do PIB em estrutura social e Coeficiente de Gini) só incluem os seis países restantes.
O estudo apontou também que a proporção da população centro-americana continental que utiliza fontes de água potável melhoradas é de $93.8 \%$ (somente inferior em Nicarágua; $87 \%$ ) e que a proporção da população que utiliza instalações sanitárias melhoradas é $78.4 \%$, não sendo atingida pelas nações de Guatemala (63.9\%), Nicarágua (67.9\%), Panamá (75\%) e El Salvador (75\%), e com especial destaque para Costa Rica (94.5\%) e Belize (90.5\%), por expressar valores muito acima do esperado.

Tabela 02. Perfil sociossanitário da América Central Continental, segundo CEPALSTAT.

\begin{tabular}{|c|c|c|c|c|c|c|c|}
\hline País & $\mathrm{PIEPIBE}^{1}$ & PIEBIBsa ${ }^{2}$ & $\mathrm{RMM}^{1}$ & $\mathrm{CGN}^{2}$ & PPFAPM $^{3}$ & PPISM $^{3}$ & $\mathrm{PIEPIBSo}^{3}$ \\
\hline BLZ & 7.4 & 6.2 & 36.0 & - & 99.5 & 90.5 & - \\
\hline$C R C$ & 7.4 & 8.1 & 27.0 & .49 & 97.8 & 94.5 & 24.0 \\
\hline SLV & 3.8 & 6.9 & 46.0 & .41 & 93.8 & 75.0 & 14.6 \\
\hline GTM & 2.8 & 5.7 & 95.0 & .54 & 92.8 & 63.9 & 7.0 \\
\hline HND & 6.0 & 7.6 & 65.0 & .48 & 91.2 & 82.6 & 8.0 \\
\hline NCR & 4.3 & 7.8 & 98.0 & .49 & 87.0 & 67.9 & 11.1 \\
\hline PNM & 3.2 & 7.0 & 52.0 & .50 & 94.7 & 75.0 & 8.8 \\
\hline MRACC & 5.0 & 7.0 & 60.0 & .48 & 93.8 & 78.4 & 12.25 \\
\hline \multicolumn{8}{|c|}{$\begin{array}{l}\text { MRACC: Média regional da América Central Continental; BLZ: Belize; CRC: Costa Rica; SLV: El Salvador; GTM: } \\
\text { Guatemala; HND: Honduras; NCR: Nicarágua; PNM: Panamá; PIEPIBE: Proporção do investimento sob PIB em } \\
\text { educação; PIEPIBsa: Proporção do investimento sob PIB em saúde; RMM: Razão da mortalidade materna; CGN: } \\
\text { Coeficiente de Gini; PPFISM: Proporção da população que utiliza fontes de água potável melhoradas; PPISM: } \\
\text { Proporção da população que utiliza instalações sanitárias melhoradas; PIEPIBSo: Proporção do investimento estatal } \\
\text { sob PIB em social. 'Projeções 2017; }{ }^{2} \text { Projeções 2015; }{ }^{3} \text { Projeções 2018. }\end{array}$} \\
\hline
\end{tabular}

Fonte: Elaborado pelos autores (2020). 
Das informações anteriores (Tabela 01; Tabela 02), podem ser levantadas as seguintes asseverações:

1. Costa Rica e Belize foram os países da ACC que projetaram melhor perfil sociossanitário para o desenvolvimento humano de seus cidadãos (segundo índices sociais, econômicos e sanitários). No caso da Costa Rica, destacou-se que essa nação ultrapassou todos os valores esperados, em todos os aspectos estudados;

2. Guatemala, El Salvador e Honduras, mostraram maiores obstáculos para alcançarem as médias regionais, indicando que nesses países o desenvolvimento humano de seus cidadãos se encontra mais comprometido. Chamou a atenção o caso da Guatemala, pois a referida nação não conseguiu atingir a meta regional, em nenhum dos quesitos analisados nesse estudo.

O comportamento epidemiológico da COVID-19 na ACC segue o padrão mundial de tendência ascendente (Tabela 03). O primeiro caso da região foi notificado na Costa Rica, em 06 de março de 2020. No período estudado, a ACC registrou um total de 194,701 casos confirmados para COVID-19 ( $L i=03 ; \mathrm{Ls}=65,256)$. A média regional de casos confirmados em março de 2020 era de 250 notificações positivas, chegando a ser 27,814 para julho de 2020.

No início da crise sociossanitária (março), Panamá $(n=1,181 ; 68 \%)$, Costa Rica $(n=347 ; 20 \%)$ e Honduras $(n=141 ; 8 \%)$ foram os três países que registraram maior incidência de notificações positivas, respectivamente. No último mês observado (julho), Panamá ( $n=65,256 ; 33.5 \%$ ) manteve a tendência positiva de contágio mais elevada da região, ora seguidos por Guatemala $(n=49,789 ; 25.6 \%)$ e Honduras ( $n=42,014 ; 21.6 \%)$.

O coeficiente de contágio foi relativamente baixo em Belize, ao longo do período estudado, sendo que até 31 julho de 2020, esse país respondeu por apenas $0.02 \%$ do total de casos confirmados na ACC. Ademais, chama a atenção o fato desse país ter conseguido manter o coeficiente de contágio em $0.0 \%$, de abril a maio de 2020. De igual modo, cumpre ver que a Nicarágua também apresentou um coeficiente de contágio bastante baixo (1.9\%), quando comparado com o resto dos países estudados.

Tabela 03. Total de casos confirmados para COVID-19 na América Central Continental, julho 2020.

\begin{tabular}{|c|c|c|c|c|c|c|c|}
\hline País & Março & Abril & Maio & Junho & Julho & Coef. Contágio (\%) & Data $1^{\circ}$ caso \\
\hline Belize & 03Li & 18 & 18 & 24 & 48 & .02 & $23 / 03 / 2020$ \\
\hline Costa Rica & 347 & 719 & 1,056 & 3,459 & 17,290 & 8.9 & 06/03/2020 \\
\hline El Salvador & 32 & 424 & 2,517 & 6,438 & 16,632 & 8.5 & $19 / 03 / 2020$ \\
\hline Guatemala & 38 & 599 & 5,087 & 18,096 & 49,789 & 25.6 & $15 / 03 / 2020$ \\
\hline Honduras & 141 & 771 & 5,202 & 19,558 & 42,014 & 21.6 & $11 / 03 / 2020$ \\
\hline Nicarágua & 05 & 14 & 759 & 2,519 & 3,672 & 1.9 & $19 / 03 / 2020$ \\
\hline Panamá & 1,181 & 6,532 & 13,463 & 33,550 & $65,256 \mathrm{Ls}$ & 33.5 & $10 / 03 / 2020$ \\
\hline MRACC & 1,747 & 9,077 & 28,102 & 83,644 & 194,701 & 100 & $06 / 03 / 2020$ \\
\hline
\end{tabular}

Fonte: Elaborado pelos autores (2020) 
Até 31 de julho de 2020, registrou-se um total de 5,398 óbitos por COVID-19 na ACC ( $L i=0 ; \quad L s=1,924)$, como exposto na Tabela 04. A média regional de óbitos em março de 2020 era de 6 passamentos, chegando a ser 771 para julho de 2020.

Constatou-se um coeficiente de letalidade regional de $0.02 \%$, corroborando com o perfil mundial de baixa letalidade. A primeira morte causada pela doença no território centro-americano continental ocorreu na data de 11 de março de 2020, em Panamá.
Os três países com maior letalidade foram, respectivamente: Guatemala $(n=1,924 ; 35.6 \%)$, Panamá $(n=1,421 ; 26.3 \%)$ e Honduras $(n=1,337 ; 24.8 \%)$. Quando comparados com o coeficiente regional, viu-se que esses países ultrapassaram drasticamente tal estatística (0.02\%) Paradoxalmente, nos países de Belize, Nicarágua e Costa Rica, o coeficiente de letalidade da doença não chegou a alcançar 3.0\%, mostrando importante assimetria dos efeitos sociossanitários provocados pela pandemia.

Tabela 03. Total de óbitos por COVID-19 na América Central Continental, julho 2020.

\begin{tabular}{cccccccc} 
País & Março & Abril & Maio & Junho & Julho & Coef. letalidade (\%) & Data $1^{\circ}$ óbito \\
\hline Belize & $0 \mathrm{Li}$ & 02 & 02 & 02 & 02 & .04 & $06 / 04 / 2020$ \\
\hline Costa Rica & 02 & 06 & 10 & 16 & 150 & 2.8 & $19 / 03 / 2020$ \\
\hline El Salvador & 01 & 10 & 46 & 174 & 448 & 8.3 & $31 / 03 / 2020$ \\
\hline Guatemala & 01 & 16 & 108 & 773 & 1,924 Ls & 35.6 & $16 / 03 / 2020$ \\
\hline Honduras & 07 & 71 & 212 & 497 & 1,337 & 24.8 & $26 / 03 / 2020$ \\
\hline Nicarágua & 01 & 03 & 35 & 83 & 116 & 2.1 & $27 / 03 / 2020$ \\
\hline Panamá & 30 & 188 & 336 & 631 & 1,421 & 26.3 & $11 / 03 / 2020$ \\
\hline MRacc & 42 & 296 & $\mathbf{7 4 9}$ & $\mathbf{2 , 1 7 6}$ & $\mathbf{5 , 3 9 8}$ & $\mathbf{1 0 0}$ & $\mathbf{1 1 / 0 3 / 2 0 2 0}$ \\
\hline $\begin{array}{l}\text { MRAcc: Média regional da América Central Continental. Estatísticas comtemplam recorte temporal de 01 de março } \\
\text { de 2020 a 31 de julho de 2020. Li= Limite inferior; Ls= Limite superior. }\end{array}$ & & & \\
\hline
\end{tabular}

Fonte: Elaborado pelos autores (2020)

Das informações anteriores (Tabela 03; Tabela 04), podem ser levantadas as seguintes asseverações:

1. Belize, Nicarágua e Costa Rica foram os países que projetaram menor coeficiente de contágio por COVID-19 no período observado. De modo contrário, Panamá, Guatemala e Honduras projetaram coeficientes de contágio alarmantes, concentrando $80.6 \%$ do total de casos positivados;

2. Apesar do coeficiente de letalidade da COVID-19 ter sido relativamente baixo (0.02\%) na ACC, os países de Guatemala, Panamá e Honduras expressaram coeficientes de letalidade exorbitantes, uma vez que os referidos países responderam por $86.7 \%$ do total de óbitos confirmados na região.
Em observância com as informações produzidas nesse estudo, não se rejeita à hipótese de pesquisa, isto é, HO: $\mu$ = países com melhor (mais favorável) perfil de desenvolvimento humano apresentaram menor coeficiente de letalidade e de contágio pela COVID-19 durante o primeiro semestre de 2020 .

\section{DISCUSSÃO}

O primeiro caso de COVID-19 na América Latina foi registrado no Brasil, na data de 21 de fevereiro de 2020, tratando-se de um indivíduo do sexo masculino, de 61 anos de idade e com histórico de viagem internacional à Itália [11]. 
Posteriormente, o número de casos confirmados para a doença espalhou-se nesse país, de forma exponencial, alcançando a condição de crise epidêmica em todas as regiões brasileiras. Em seguida, países vizinhos também começaram a notificar o surgimento de quadros de pneumonia aguda, do que seria um vírus não conhecido pelas entidades sanitárias locais, mas que já vinha sendo alertado pela OMS $[1,6,12]$.

No caso dos países centro-americanos, nosso estudo constatou que a ACC recebeu os efeitos da pandemia de forma mais retardada, havendo sido notificado o primeiro caso 13 dias após da América do Sul (06/03/2020, na República de Costa Rica). O cenário sociossanitário provocado pela COVID-19, permite entender que os sanitaristas epidemiologistas, e todos os profissionais da saúde, em geral, devem trabalhar em conjunto e utilizar os recursos digitais no seu melhor, e isto deve ser aumentado, especialmente entre as pessoas que vivem em áreas de difícil acesso [11,12]. Como cada país da América Latina depende de diferentes mecanismos de financiamento para combatê-la e tem diferentes comportamentos culturais, experiências passadas bem-sucedidas são quintessenciais. O ponto mais crucial é a identificação de ações de implementação bem-sucedidas de controle de surtos no passado e números sobre como adaptá-lo às necessidades da COVID-19 [13].

Outrossim, tem-se tornado visível que a pandemia vislumbrou os já conhecidos entraves dos sistemas de saúde e de assistência social, que caracterizam à maioria dos países centro-americanos. Importante estudo, que abordou os efeitos da COVID-19 na América Latina, apontou que a atual pandemia é outro lembrete da necessidade imperativa de um compromisso intensificado e sustentado com a concepção de protocolos de resposta baseados em evidências, que, de forma transdisciplinar e multissetorial, podem alcançar a redução do número de vidas perdidas e para responder às dificuldades sociais, ambientais, políticas e econômicas geradas por essas pandemias [14]. Nesse ângulo apreensivo, torna-se de suma importância que a região centro-americana fortaleça políticas emergenciais de saúde pública de importância internacional, com simulações em todos os países, e que o trabalho sobre as fronteiras territoriais seja reforçado a fim de identificar fatores de risco através de pesquisas direcionadas ao combate e prevenção da COVID-19 [15].

O cenário sociossanitário da crise atual de Saúde Pública na ACC tem de ser analisado e visto com a devida cautela. Percebe-se, por exemplo, que a pandemia da COVID-19 chegou de forma tardia nos países centro- americanos, quando comparada com o resto de países da América Latina. Isto permitiu que a maioria desses países <centro-americanos> adotasse um comportamento preventivo perantes os diversos desafios impostos pela doença. O estudo de Alvarez e Harris, que descreveu os casos acumulados por COVID-19 no Continente Americano (regiões Norte, Central e Sul) mostrou que até março de 2020 os países da ACC responderam por apenas $0.97 \%$ do total de contágios por SARS-CoV-2 (1,370; $140,747)$ nesse mês. De forma contrária, as regiões norteamericana e sul-americana têm projetado um impacto mais negativo, em relação aos coeficientes de contágio e de letalidade [16].

No entanto, a repercussão do contexto sociossanitário se vê de forma mais acentuada em Panamá, Guatemala e Honduras, como exposto nas Tabelas 03 (coeficiente de contágio) e 04 (coeficiente de letalidade). Nessa compreensão, Loiza et al., acenam para os entraves não só simplesmente assistenciais, pois incluem também os âmbitos técnicos e organizacionais dos sistemas públicos de saúde centro-americanos. Na visão dos autores, "devemos permanecer calmos, reconhecendo cuidadosamente as lições aprendidas de outras regiões para evitar vítimas humanas" $[13,17]$. Conquanto, tal calmaria não quer dizer, de forma específica, apequenar os esforços e/ou minimizar a relevância do panorama regional. Trata-se, no sentido amplo, da perspectiva de integração regional, no objetivo final de promover a maior qualidade de assistência sanitária e condições de vida para que os cidadãos centroamericanos consigam enfrentar a crise pandêmica.

Seria pouco sensato pensar que a ACC está preparada para enfrentar uma crise de Saúde Coletiva com tal magnitude. Fato é que, com o esquema de contágio internacional e a rápida propagação do SARSCoV-2, as medidas de vigilância sanitária em região de fronteira mereceram destaque, pois os países precisaram (e ainda o fazem), de reforçar as intervenções nesses espaços geográficos. Tal-qual efeito dominó, um a um foi estipulando instrumentos jurídico-sanitários, que foram ao encontro das recomendações divulgadas por organizações internacionais [18]. Isto porque emergências higiênicas registradas no século passado, já nos permitiram entender que "o movimento de pessoas, animais e bens são componentes importantes na distribuição de doenças infecciosas no mundo" [19]. Igualmente, serve lembrar que as regiões de fronteira são "estratégicas para o controle de agravos, o desenvolvimento de políticas e ações direcionadas a nível nacional e/ou regional" [19].

O posicionamento dos governos perante a perspectiva 
de soberania sanitária centro-americana, pode ser modelo para outras regiões latino-americanas e, de forma mais pretensiosa, para o resto do mundo. Em 12 de março de 2020, foi celebrada reunião com os Chefes de Estado e os Ministros de Saúde Pública da ACC, com o objetivo de discutir os impactos projetados para a região e pensar as estratégias de articulação e atuação, no âmbito do Consejo de Ministros de Salud de Centroamérica y República Dominicana. Como resultado, foi promulgado o Plano de Contingência Regional do Sistema de Integración Centroamericana - SICA [20], o qual foi estruturado em três eixos principais, a saber: 1) saúde e gestão de riscos; 2) finanças e comércio e 3) segurança, justiça e migração. Outrossim, foram considerados dois eixos transversais: 1) comunicação estratégia e 2) gestão da cooperação internacional.

No tangente ao primeiro eixo, saúde e gestão de riscos, foram estabelecidas as medidas de prevenção e contenção, medidas de manejo de pacientes em cada tipo de casos (suspeita, reagentes e não-reagentes); harmonização de mensagens informativas, preventivas e educativas; o acesso a medicamentos, dispositivos médicos e outros bens de interesse sanitário; mecanismo regional para fortalecer a capacidade de preparação, mitigação, resposta e assistência humanitária [20]. De forma especial, faz-se jus mencionar que as medidas de prevenção e preparação regional contra a COVID-19 relevam:

\footnotetext{
Monitoramento das condições de risco - social, econômico e ambiental de grupos, comunidades e áreas mais expostas e vulneráveis, a fim de fortalecer sua resiliência, atualizando ou desenvolvendo planos específicos de gestão de risco (...) desenvolver medidas de prevenção e de preparação para a gestão e o cuidado de emergências geradas por outros perigos (enchentes, terremotos incêndios, entre outros), que coincidem com a fase de cuidado e recuperação da pandemia (...) consulta e troca de experiências com países que começaram antes com quarentenas ou processos de isolamento social, como China, Itália e Espanha e (...) identificação e promoção de mecanismos de gestão de risco financeiro, que podem proteger e fortalecer a subsistência da população mais vulnerável, bem como do setor privado [20:18]
}

De outro lado, as informações produzidas neste estudo não devem ser entendidas de forma estática. Diversas pesquisas devem continuar a serem desenvolvidas, pois reconhece-se que o comportamento epidemiológico do vírus pode mudar drasticamente em questão de semanas. Não obstante, estudos têm apontado que, se bem a frequência absoluta de óbitos por COVID-19 e de infecções por SARS-CoV-2 continua a crescer, os países centro-americanos têm mantido certos padrões, onde, por exemplo, Belize, Nicarágua e Costa Rica, têm-se caracterizado por possuírem menores coeficientes de contágio e letalidade, contrapondo as realidades observadas em Guatemala, Panamá e Honduras [21]. Outrossim, tal asseveração se tornou ainda mais interessante para o presente estudo, uma vez que os países mais afetados pelo coronavírus, foram justamente aqueles que mostraram perfis sociossanitários mais precários, em todos as áreas observadas: sanitária, educativa, econômica e social.

Finalmente, mencionar que este estudo apresentou algumas limitações. Por tratar-se de uma situação de emergência sanitária que apresenta flutuações diárias (coeficientes de contágio e letalidade), as projeções estatísticas produzidas nesse trabalho não devem ser generalizadas, uma vez que os dados foram incluídos apenas para o primeiro semestre de 2020. Além disso, devese informar que as estatísticas fornecidas incluíram apenas indivíduos testados com resultados reagentes (positivos), e que a extração de variáveis sociodemográficas, como: sexo, raça, nacionalidade, idade, faixa-etária, não foi possível, uma vez que o site consultado disponibiliza informações brutas, sem estratificação pelas referidas variáveis.

Conflitos de interesse: Não existem conflitos de interesse a declarar.

\section{REFERÊNCIAS}

1. Sun $P, L u X, X u C$, Sun $W$, Pan B. Understanding of COVID-19 based on current evidence. J Med Virol. 2020. https://dx.doi.org/10.1002/imv.25722

2. Sallar E, Lescure FX, Yazdanpanah $Y$, Mentre F, PeifferSmadja N. Type 1 interferons as a potential treatment against COVID-19. Antiviral Research. 2020;1-3. https://dx.doi.org/10.1016/j.antiviral.2020.104791

3. Liu K, Chen $Y$, Lin R, Han K. Clinical features of COVID-19 in elderly patients: a comparison with young and middle-aged patients. J Infect Prev. 2020;80(1)1418.

https://dx.doi.org/10.1016/j.jinf.2020.03.005

4. Comissão Econômica para a América Latina e o Caribe - CEPAL. América Latina y el Caribe: actualización de proyecciones de crecimiento 2020. 
5. Gutiérrez-Murillo RS, Gamarra CJ, Svoboda WK, Svoboda NK. O suicídio como problema de saúde coletiva na América Central Continental: uma análise dos casos consumados entre 2010-2016. RELA Cult. 2020;6(1)123. https://dx.doi.org/10.23899/relacult.v6i1.1781

6. Delgado BM, Silva AP, Rodríguez JM. Mapa conceptual de salud y propiedad intelectual en Centroamérica y República Dominicana. Revista Panamericana de Salud Pública. 2019;43(1)1-9. $\quad$ https://dx.doi.org/10.26633/RPSP.2019.4

7. Só Geografia. Virtuous Tecnologia da Informação, 2007-2020. América Central. 2020. [Cited 08/22/2020]. Disponível em: http://www.sogeografia.com.br/ Conteudos/Continentes/America/content18.php

8. Comissão Econômica para a América Latina e o Caribe CEPAL. Bases de Dados e Publicações Estatísticas. [internet]. 2020. [Cited 08/22/2020]. Disponível em: https://estadisticas. cepal.org/cepalstat/perfilesNacionales.html?idioma=spanish

9. University of Washington School of Medicine. Institute of Health Metrics and Evaluation - IHME. COVID-19 Projections. [internet]. 2020. [Cited 08/22/2020]. Disponível em: http://www.healthdata.org/covid/updates

10. Pereira SD. Conceitos e definições da saúde e epidemiologia usados na vigilância sanitária. [internet]. 2004,1-22. [Cited 08/22/2020]. Disponível em: http://www.cvs.saude.sp.gov.br/pdf/epid visa.pdf

11. Rodríguez-Morales AJ et al. COVID-19 in Latin America: The implications of the first confirmed case in Brazil. Travel Medicine and Infectious Disease. 2020;35(1)13. https://dx.doi.org/10.1016/j.tmaid.2020.101613

12. Silva RF, Pitzurra R. What are the factors influencing the COVID-19 outbreak in Latin America? Travel Medicine and Infectious Disease. 2020;35(1)12. $\quad$ https://dx.doi.org/10.1016/j.tmaid.2020.101667

13. Ramírez-Paulino $R$, Tapia $L$. Learning from Pandemics in the Americas: The Dominican Republic Programmatic Response Against a novel Coronavirus (COVID-19). InterAmerican. 2020;3:e202003024. https://dx.doi.org/10.31005/iajmh.v3i0.104

14. Sánchez-Duque J, Arce-Villalobos LR, Rodríguez-
Morales AJ. Enfermedad por coronavirus 2019 (COVID-19) en América Latina: papel de la atención primaria en la preparación y respuesta. Atención Primaria. 2020;53(6):369372. $\quad$ https://dx.doi.org/10.1016/j.aprim.2020.04.001

15. Rodríguez-Morales AJ. Preparación y control de la enfermedad por coronavirus 2019 (COVID-19) en América Latina. Acta Med Peruana. 2020;37(1):37. https://dx.doi.org/10.35663/ amp.2020.371.909

16. Alvarez-Pierre R, Harris PR. COVID-19 en América Latina: retos y oportunidades. Rev Chilena Pedia. 2020;91(2):179182. $\quad$ http://dx.doi.org/10.32641/rchped.vi91i2.2157.

17. Loiza RJ, Kosagisharaf R, Eskildsen GA. COVID-19 pandemic in Panama: lessons of the unique and risks and research opportunities for Latin America. PAJPH. 2020; 44:1-5. https://dx.doi.org/10.26633/RPSP.2020.86

18. Hardiman $M$, Wilder-Smith $A$. The revised international health regulations and their relevance to travel medicine. J Travel Med. 2007; 14(3):141-144. https://dx.doi.org/10.1111/j.1708-8305.2007.00117.x

19. Bruniera-Oliveira R, Horta MAPP, Belo VSS, Carmo EH, Verani JFS. Desenvolvimento da vigilância epidemiológica de fronteira no contexto da globalização: conceitos e marco teórico. Tempus Actas em Saúde Coletiva. 2014; 8(3):75-93. https://dx.doi.org/10.18569/tempus.v8i3.1565.

20. Sistema de Integración Centroamericana - SICA. Secretaria de Estado del Consejo de Ministros de Salud de Centroamérica y República Dominicana. Plan de Contingenica Regional: orientado a complementar los esfuerzos nacionales para la prevención, contención y tratamiento del COVID-19.

21. Gutiérrez Murillo RS, Gamarra CJ. Intervenciones salubristas adoptadas por la República de Costa Rica durante la epidemia de la COVID-19. Rev Cubana Salud Pública. 2020;46(4):e2554. 